\title{
Angiogenic endothelium-specific nestin expression is enhanced by the first intron of the nestin gene
}

\author{
Masanori Aihara ${ }^{1,2}$, Ken-ichi Sugawara ${ }^{2}$, Seiji Torii ${ }^{1}$, Masahiro Hosaka ${ }^{1}$, \\ Hideyuki Kurihara ${ }^{2}$, Nobuhito Saito ${ }^{2}$ and Toshiyuki Takeuchi ${ }^{1}$ \\ ${ }^{1}$ Department of Molecular Medicine, Institute for Molecular and Cellular Regulation, Gunma University, \\ Maebashi, Japan and ${ }^{2}$ Department of Neurosurgery, Gunma University Graduate School of Medicine, \\ Maebashi, Japan
}

\begin{abstract}
Nestin is a member of intermediate filaments abundantly expressed in neural stem cells and glioblastomas. The nestin gene has four exons and three introns, and neural cell-specific expression is regulated by the second intron. We previously reported that nestin was invariably detected in the tumor endothelium in gliomas even though tumor cells were negative for nestin. In the present study, we further confirmed nestin immunostaining in tumor endothelium of a variety of common cancers, including lung, stomach, colon, and cervical carcinomas. We examined an endothelium-specific regulator using human umbilical vein endothelial cells (HUVECs) and human glioblastoma-derived U251 cells. In a luciferase reporter assay, the first intron plus $5^{\prime}$ upstream promoter ( $5^{\prime}$ UP) gave the highest activity, followed by $5^{\prime}$ UP, and the second intron plus $5^{\prime}$ UP. However, the assay values were much lower by HUVEC extracts than by U251 cell extracts. Although green fluorescent protein expression was positive over all U251 cells under either the first intron, second intron, or ubiquitously active CAG promoter, the fluorescence in HUVECs was limited to a few cells even under the first intron. This difference came from the growth feature of HUVECs which exhibit growth arrest by contact inhibition. We found that the nestin expression was specific to proliferative endothelium, by using proliferation markers in hemangioblastomas and in situ hybridization. Using an endothelial tube formation assay, tyrosine kinase domain-deleted VEGF receptor KDR effectively abolished the tube formation under the first intron. We suggest that the nestin expression in tumor endothelium is enhanced by the first intron.
\end{abstract}

Laboratory Investigation (2004) 84, 1581-1592, advance online publication, 25 October 2004; doi:10.1038/labinvest.3700186

Keywords: nestin; tumor angiogenesis; intronic enhancer; glioblastoma; hemangioblastoma; endothelial cells

Nestin is a member of intermediate filaments and found abundantly in neuroepithelial stem/progenitor cells. ${ }^{1,2}$ Nestin forms intermediate filament bundles with other intermediate filaments such as vimentin and glial fibrillary acidic protein (GFAP) by copolymerization in neuroepithelial cells. ${ }^{3}$ In copolymerized intermediate filaments, nestin induces disassembly of the vimentin filaments to nonfilamentous aggregates or particles in mitotic cells, suggesting that nestin plays a role in the distribution of vimentin filaments to daughter cells during progenitor cell division. ${ }^{4}$ Nestin transgene-

Correspondence: Dr T Takeuchi, MD, Department of Molecular Medicine, Institute for Molecular and Cellular Regulation, Gunma University, Showa-machi, Maebashi 371-8512, Japan.

E-mail: tstake@showa.gunma-u.ac.jp

Received 27 June 2004; revised 14 August 2004; accepted 16

August 2004; published online 25 October 2004 promoted $\beta$-galactosidase expression analysis in mice has revealed LacZ activity in neuroepithelium and somites shortly after neural tube closure (E9). ${ }^{5}$ With this neural stem/progenitor cell-specific expression, nestin has been utilized to harvest neuronal progenitor cells from embryonal stem cells. ${ }^{6}$ Furthermore, significant levels of nestin have been detected in human gliomas. ${ }^{7,8}$

Recently, we re-evaluated the nestin expression in 71 cases of brain tumors including 40 cases of gliomas that ranged from low to high malignancy grades. ${ }^{9}$ Nestin was highly immunostained in gliomas with a higher malignant grade. High levels of nestin have also been detected in tumor endothelium in glioma tissues, irrespective of its expression in tumor cells. Furthermore, high levels of nestin have been detected in nonneoplastic, primarycultured bovine aortic endothelial cells in static culture, but nestin was not detected when the 
endothelial cells underwent shear stress flow, during which endothelial cells exhibit differentiated features and a lower rate of growth. ${ }^{9,10}$

Nestin mRNA is approximately 6.2 kilobases $(\mathrm{kb})$ long and its gene consists of four exons and three introns. ${ }^{5}$ Neuroepithelium-specific nestin expression is not driven by a conventional $5^{\prime}$ upstream promoter ( $\left.5^{\prime} \mathrm{UP}\right)$, but driven by the second intron of the nestin gene. ${ }^{2,5,11}$ The neuroepithelium-specific enhancer region in the approximately $1.7 \mathrm{~kb}$ rat second intron has been narrowed down to $636 \mathrm{bp}$ in rats and $714 \mathrm{bp}$ in humans. ${ }^{2,11}$ The neuroepitheliumspecific regulator, consisting of the second intron before the $2.1 \mathrm{~kb}$-long $5^{\prime}$ UP region of the gene, drove LacZ expression in parallel with the extent of mRNA expression in seven human glioma cell lines. ${ }^{8}$ However, in terms of nestin expression in angiogenic endothelium, a regulatory region has not yet been identified.

Endothelial cells are derived from hemangioblasts, which can develop into both hematopoietic precursor cells and angioblasts (pre-endothelial cells). ${ }^{12}$ Hemangioblasts are characterized by the hematopoietic marker proteins CD34, and VEGF receptor type 2 (also called Flt-1, or KDR). ${ }^{12,13}$ In tumor angiogenesis, bone marrow-derived endothelial progenitor cells contribute much to newly forming angiogenic vessels. ${ }^{12,14}$ Thus, in the angiogenic endothelium of tumors, a significant number of hemangioblast-like hematopoietic precursor cells may be integrated in the endothelial cell population. We presume that nestin, together with CD34 and KDR, may display quite specific expression in the angiogenic epithelium contained in common tumors.

In the present study, we confirmed nestin expression in tumor epithelium in a variety of common cancers including lung, stomach, colon, and cervical cancers. By overcoming the growth arresting feature of human umbilical vein endothelial cells (HUVECs), we identified an angiogenesis-specific regulator in the first intron of the nestin gene.

\section{Materials and methods}

\section{Tumor Tissues}

We used 82 common tumors and eight hemangioblastomas. The 82 common tumors included eight lung cancers, nine esophageal cancers, 20 gastric cancers, 22 colorectal cancers, two hepatocellular carcinomas, 15 uteric cancers, and six ovarian cancers, as listed in Table 1. Eight hemangioblastomas were localized solitarily in the cerebellum. All tumors were resected at the Gunma University Hospital. Uterine tissue was obtained from a patient who had undergone radical hysterectomy for ovarian cancer and brain tissue was procured from a gross-total glioma resection from the Departments of Obstetrics and Gynecology and Neurosurgery, respectively.

\section{Cell Culture}

HUVECs were isolated from term umbilical cord veins by flowing a $0.1 \%$ collagenase solution up and down the vein using syringes fixed at both ends of the cord. The procedure was approved by the Ethics Committee of the Gunma University. The isolated HUVECs were grown to subconfluent levels in RPMI medium with $20 \%$ fetal bovine serum and $100 \mu \mathrm{g} / \mathrm{ml}$ endothelial growth supplement (Harbor Bio-products, Northwood, MA, USA), and $10 \mathrm{U} / \mathrm{ml}$ heparin. For nestin-positive control cells, we used the U251 human glioblastoma cell line. ${ }^{8}$

A tube formation assay with HUVECs was performed on Matrigel layer, ${ }^{15}$ which is a crude extract of basal membrane from mouse Engelbreth-HolmSwarm tumors. Briefly, Matrigel (400 $\mu$ l) (Collaborative Biomedical Products, Medford, MA, USA) was placed on a $3.0 \mathrm{~cm}$ culture plate and left for polymerization at $37^{\circ} \mathrm{C}$ for $1 \mathrm{~h}$. HUVECs were seeded at $3 \times 10^{5}$ cells/plate, and cultured for an indicated time to form a tubular network.

\section{Morphological Studies}

To evaluate cell proliferation, we used two proliferation markers, Ki-67 and PCNA, which appear to reflect different proliferative modes. ${ }^{16}$ The antibody to human nestin was prepared in rabbits as described previously. ${ }^{8}$ We obtained mouse monoclonal antibodies to human von Willebrand factor (vWF) and human Ki-67 (MIB-1) from DAKO (Glostrup, Denmark), mouse monoclonal antibodies to KDR and proliferating cell nuclear antigen (PCNA) from Santa Cruz Biotechnology, Inc. (Santa Cruz, CA, USA), and mouse monoclonal antibody to human CD34 from Nichirei (Tokyo, Japan).

Tumor tissues were fixed in $4 \%$ paraformaldehyde in $0.1 \mathrm{M}$ phosphate buffer, $\mathrm{pH} 7.4$ for $24 \mathrm{~h}$. The tissue sections were first incubated overnight at $4^{\circ} \mathrm{C}$ with the primary antibody to nestin at a dilution of 1:7500, vWF at 1:200, KDR at 1:200, CD34 at 1:1000, PCNA at 1:5000, Ki-67 at 1:5000. An LSAB2/HRP staining kit (DAKO) was used as a secondary antibody reaction system. The procedure consisted of a secondary antibody reaction followed by an enzyme reaction with a horseradish peroxidaselabeled streptavidin system. In the enzyme reaction, the peroxidase catalyzes 3-amino-9-ethylcarbazole to an insoluble brown-colored product. For double immunofluorescent visualization, the secondary antibody used was indodicarbocyanide (Cy3)-conjugated affinity-purified donkey anti-mouse IgG (red-colored) and fluorescein isothiocyanate (FITC)-conjugated donkey anti-rabbit IgG (greencolored) (Jackson ImmunoResearch).

\section{Luciferase Assay}

A luciferase assay was performed using the DualLuciferase Reporter Assay System (Promega, Madi- 
son, WI, USA). HUVECs or U251 cells were seeded initially at $5 \times 10^{4}$ cells/well in a 24 -well dish, and cultured for $24 \mathrm{~h}$. Lipofection with Lipofectamine (Invitrogen, Carlsbad, CA, USA) was performed with each of the seven luciferase gene constructs, including a regulator-less gene. At $6 \mathrm{~h}$ after the lipofection, the medium was changed to a new one and the culture was continued for another $18 \mathrm{~h}$. Then, the cells were harvested to prepare cell lysates using the cell lysis buffer supplied with the Assay System.

The six luciferase gene constructs were made by placing a nestin gene 'regulator' before the luciferase gene. We initially cloned the $8 \mathrm{~kb}$ rat nestin genomic DNA from a rat genomic DNA library (GIBCO/BRL, Rockville, MD, USA) using oligonucleotides based on the rat nestin cDNA sequence. ${ }^{11}$ To make chimeric nestin regulators, we cut out the $2.1 \mathrm{~kb}$ nestin basic promoter (NP) region by Xmnl, as described before. ${ }^{8}$ Then we cut out the $1.3 \mathrm{~kb}$ first intron (1i) DNA fragment by BamHI and $A c c \mathrm{I}$, and the $1.8 \mathrm{~kb}$ second intron (2i) fragment by BstYI. The 1i DNA fragment contains the $967 \mathrm{bp}$ first intron, the $283 \mathrm{bp}$ first exon, and the $37 \mathrm{bp}$ second partial exon. The 2i DNA fragment contains the $1669 \mathrm{bp}$ second intron, the $70 \mathrm{bp}$ partial second exon, the $72 \mathrm{bp}$ third exon, and the $32 \mathrm{bp}$ partial third intron. Both the $1 \mathrm{i}$ and $2 \mathrm{i}$ DNA fragments were placed before the $5^{\prime}$ upstream NP (1iNP and 2iNP). The 1i DNA fragment was half-split by PCR amplification to $370 \mathrm{bp}\left(5^{\prime} 1 \mathrm{i}\right)$ and $598 \mathrm{bp}\left(3^{\prime} 1 \mathrm{i}\right)$, and both fragments were also placed before the NP ( $5^{\prime} 1 \mathrm{iNP}$ and $\left.3^{\prime} 1 \mathrm{iNP}\right)$. Conversely, two 1i DNA fragments were placed in a tandem manner before the NP. The six nestin regulators were integrated into the firefly luciferase reporter gene vector PicaGene Basic Vector 2 (Toyo Ink Corp., Tokyo, Japan).

\section{Construction of a Nestin Regulator-Promoted Target Gene Expression System Using Adenovirus Vectors}

We used a double-adenoviral infection method that enhances weak cell/tissue-specific gene expression approximately 50-fold. ${ }^{8}$ This method uses two adenovirus vectors, a regulatory vector with the Cre recombinase under a tissue-specific regulator, and an effecter vector comprises of the CAG promoter. We used an effecter gene interrupted with a loxP site-flanked stuffer gene at both ends for Cre excision. The CAG promoter, derived from the cytomegalovirus immediate-early region enhancer followed by a chicken $\beta$-actin promoter plus the $\beta$ globin $3^{\prime}$-flanking sequence, is one of the strongest promoters in eukaryotic cells. ${ }^{17}$

The three nestin regulators (NP, 1iNP, and 2iNP) were placed before the Cre recombinase sequence in a cosmid pAxAwNCre (Riken Gene Bank). ${ }^{8}$ The cosmid constructs were subjected to a homologous recombination with the Eco T221-digested DNAterminal protein complex of Ad5-dlX in HEK 293
Table 1 Summary of nestin immunostaining in 82 common tumors

\begin{tabular}{|c|c|c|c|c|}
\hline \multirow[t]{2}{*}{ Cancer type } & \multirow[b]{2}{*}{$\mathrm{n}$} & \multicolumn{3}{|c|}{ Intensity } \\
\hline & & 0 & $1+$ & $2+$ \\
\hline \multicolumn{5}{|l|}{ Lung cancer } \\
\hline Adenocarcinoma & 7 & 3 & 3 & 1 \\
\hline Small cell carcinoma & 1 & 1 & 0 & 0 \\
\hline \multicolumn{5}{|l|}{ Esophageal cancer } \\
\hline Squamous cell carcinoma & 9 & 0 & 4 & 5 \\
\hline \multicolumn{5}{|l|}{ Gastric cancer } \\
\hline Adenocarcinoma & 19 & 4 & 10 & 5 \\
\hline Malignant lymphoma & 1 & 0 & 1 & 0 \\
\hline \multicolumn{5}{|l|}{ Colon cancer } \\
\hline Adenocarcinoma & 22 & 7 & 5 & 10 \\
\hline \multicolumn{5}{|l|}{ Hepatoma } \\
\hline Hepatocellular carcinoma & 2 & 0 & 1 & 1 \\
\hline \multicolumn{5}{|l|}{ Uteric cancer } \\
\hline \multicolumn{5}{|l|}{ Cervical } \\
\hline Adenocarcinoma & 3 & 0 & 1 & 2 \\
\hline Squamous cell carcinoma & 4 & 0 & 3 & 1 \\
\hline \multicolumn{5}{|l|}{ Corpus } \\
\hline Adenocarcinoma endometrioid & 7 & 1 & 3 & 3 \\
\hline Carcinosarcoma & 1 & 0 & 1 & 0 \\
\hline \multicolumn{5}{|l|}{ Ovarian cancer } \\
\hline Adenocarcinoma endometrioid & 5 & 1 & 2 & 2 \\
\hline Mesodermal mixed tumor & 1 & 0 & 0 & 1 \\
\hline Total & 82 & 17 & 34 & 31 \\
\hline
\end{tabular}

Nestin-stained endothelial cells were scored in one microscopic field. 0 , no staining.

$1+$, positive cells found by $\times 200$ magnification.

$2+$, positive cells found by $\times 100$ magnification.

The percent of tumors with nestin-positive endothelia was calculated as follows: $34+31 / 82=79 \%$.

cells. The regulator adenovirus vectors (Ad1i NPNCre, Ad2iNPNCre, and AdNPNCre) were increased for purification by velocity density-gradient centrifugation. As an effecter adenovirus vectors, we integrated two effecter genes, GFP and $\triangle \mathrm{KDR},{ }^{18}$ behind the stuffer sequence in a cosmid pAxCAL.

\section{Western Blot Analysis}

HUVEC or U251 cell lysates were electrophoresed on a $9 \%$ polyacrylamide gel under a reducing condition, then blotted onto a nitrocellulose membrane for probing with nestin antibody (1:7500) or KDR antibody (1:5000). Blots were detected utilizing an ECL detection system (Amersham, Buckinghamshire, UK).

\section{In Situ Hybridization}

In situ hybridization for nestin was performed with the DIG RNA Labeling Kit (Boehringer Mannheim, 
Germany). To obtain template cDNAs, total RNA was isolated from HUVECs, and first-strand cDNA was prepared by reverse transcription. RNA probes were prepared in a reaction mixture containing digoxigenin-labeled UTP and other NTPs from the template cDNA with T3 RNA polymerase for an antisense-strand probe and T7 RNA polymerase for a sense-strand probe. The RNA probes were heatdenatured before use.

HUVECs fixed on a glass slide were treated with hydrogen peroxide for $15 \mathrm{~min}$ at room temperature to remove reactive oxygen, and then digested with proteinase $\mathrm{K}$ at $1 \mu \mathrm{g} / \mathrm{ml}$ at $37^{\circ} \mathrm{C}$ for $10 \mathrm{~min}$. The samples were incubated with a hybridization solution containing sense- or antisense-strand cRNAs at a concentration of $500 \mathrm{ng} / \mathrm{ml}$ at $55^{\circ} \mathrm{C}$ overnight, then subjected to a second reaction with an antidigoxygenin antibody complexed with alkaline phosphatase (Boerhinger Mannheim) at a dilution of 1:1000 for $1 \mathrm{~h}$. RNA probes were visualized with an alkaline phosphatase reaction using 5-bromo-4chloro-3-indolyl phosphate and 4-nitroblue tetrazolium chloride.

\section{Results}

\section{Nestin Localization in Common Tumors and Uterus}

We examined nestin immunostaining in 82 various common tumors, as listed in Table 1 . The nestin staining was limited only to endothelial elements and was negative in tumor cells (Figure 1a-f). Furthermore, its expression was irrespective of the carcinoma cell type: squamous carcinoma (Figure 1b) or adenocarcinoma (Figure 1c). We then examined the colocalization of CD34 that has a transmembrane domain and cytosol-residential nestin in cervical cancer (Figure 1e) and ovarian cancer (Figure 1f). We noted colocalization of both proteins in the same endothelial components (yellow staining), although a few endothelial cells stained exclusively for CD34 (red staining) or for nestin (green staining) (Figure $1 \mathrm{~g}$ and $\mathrm{h}$ ), indicating that their expression mechanisms may not be identical. Interestingly, Kim et $a l^{19}$ has demonstrated that in gastric adenocarcinomas, neovascularizing microvessel area expresses nestin more widely and densely than CD34. Among 82 tumor samples, 65 samples contained nestin-positive intratumor endothelium (79\%, Table 1).

In the human body, active angiogenesis occurs in the endothelium of the uterine endometrium and myometrium, in which the formation and destruction of neoangiogenic vessels repeat with the menstrual cycle. Indeed, nestin was positive in the endothelium of the endometrium and myometrium (Figure 1i and j, respectively). Nestin-positive cells were clustered under the glandular epithelial cell layer in the endometrium (Figure 1i). Thus, nestin must play an important role in neoangiogenesis in tumor formation and in uterine endometrium and myometrium reformation.

\section{Nestin Expression in Hemangioblastomas}

Hemangioblastoma consists of at least three cell types; mature-type endothelial cells, microcapillarylike cells, and stromal cells which are thought to be tumorigenic by expressing mutated pVLH protein. ${ }^{20}$ The origin of stromal cells has been controversial because the cells exhibit more or less endothelial, mesenchymal, or neuronal features. ${ }^{21}$ Among these cell types, nestin is localized to the microcapillary region (Figure 2a), but not to the mature-type endothelial component, which is positive for vWF (Figure 2d, arrowhead). This region appears to consist of hemangioblast-like cells because CD34 and KDR were also positive in addition to nestin (Figure $2 \mathrm{~b}$ and $\mathrm{c}$ ). ${ }^{13}$

We examined the cell proliferative features of this region using two proliferation markers; PCNA and Ki-67. PCNA-positive nuclei were distributed in numerous cells in this microcapillary region (Figure 2e), whereas Ki-67-positive cells were few and limited mostly to the microcapillary endothelia (Figure 2f). Red-colored Ki-67 was detected mostly in green-colored nestin-positive endothelial cells in the microcapillary region (Figure $2 \mathrm{~g}$ ), whereas it was also negative in nestin-negative endothelial cells (Figure 2h, arrowheads). Although the tumorigenic component of hemangioblastomas is ascribed to stromal cells, the mitotic rate with the Ki-67 index is reportedly less than $1 \% .^{20,21}$ Indeed, few Ki-67positive foamy stromal cells were detected. Thus, active replication is carried out mostly in Ki-67positive microcapillary cells. Ki-67 may disappear soon after cell replication, while PCNA proteins remain for some time in the nuclei. ${ }^{16}$ Nestin appears to have a similar lifetime to PCNA based on its similar microcapillary distribution.

\section{Angiogenic Endothelium-Specific Regulators for Nestin Expression}

Nestin was expressed strongly in HUVECs in a static culture by Western blotting (Figure 3a). We initially compared four nestin regulator constructs (regulator-less construct, 1iNP, 2iNP, and NP: Figure 3b) using a luciferase reporter assay. In HUVECs, 1iNP showed the highest activity, followed by NP (Figure 3c, left panel). We further examined another three nestin regulators (5'1iNP, $3^{\prime} 1$ iNP, and 1i1iNP: Figure 3b). However, none of these regulators exceeded their original 1iNP activity, although the $3^{\prime} 1 \mathrm{iNP}$ was highest (Figure 3d, left panel). For reference, we used U251 cells, in which a regulator with the $2 \mathrm{i}$ DNA reportedly shows high activity. ${ }^{8}$ The activities of both 1iNP and 2iNP were similarly high, and NP also showed relatively high activity (Figure 3c, right panel). Among these regulators, the $3^{\prime} 1$ iNP was 

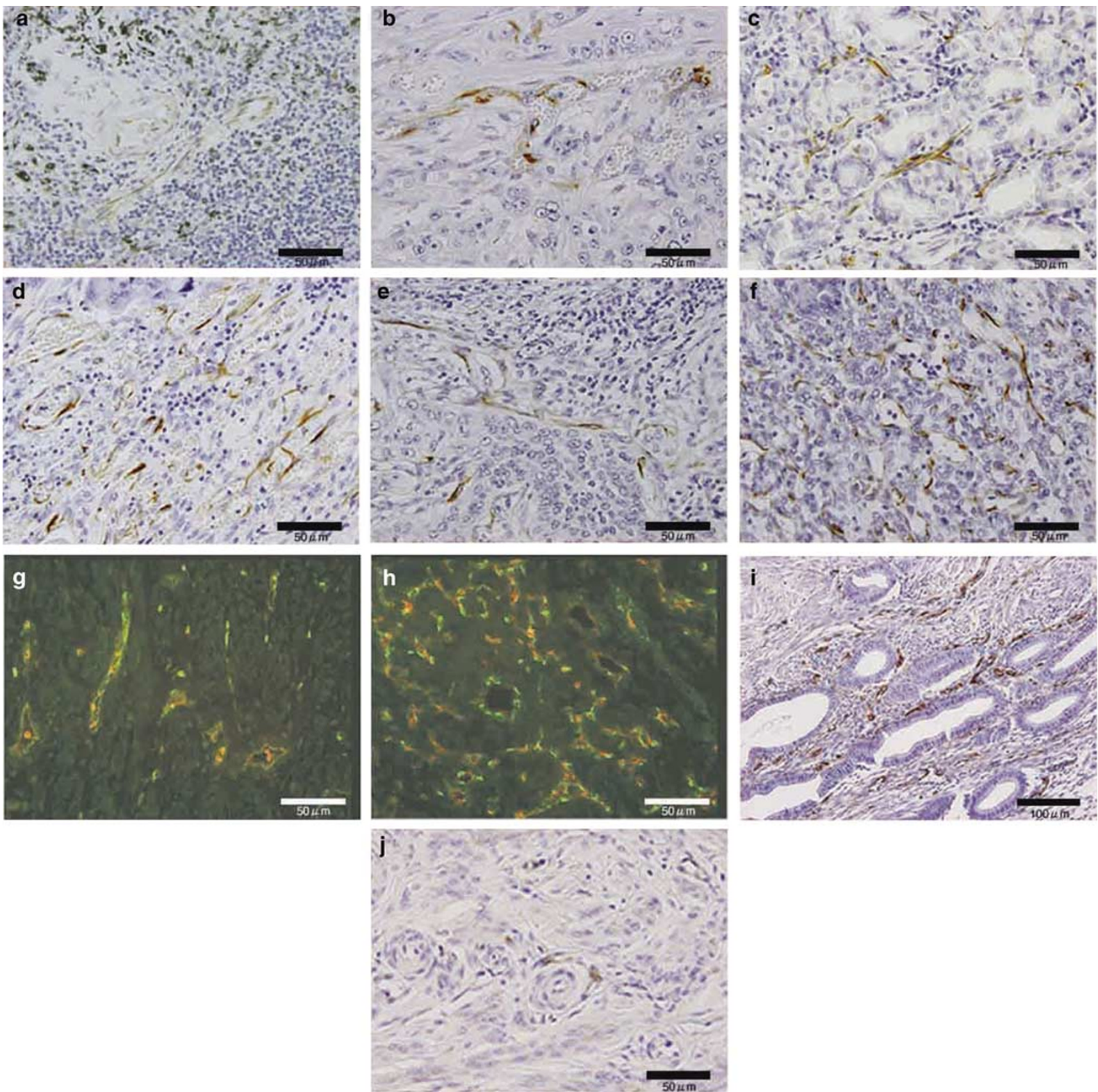

Figure 1 Nestin localization in common tumors and uterus. (a) lung cancer; (b) esophageal cancer; (c) gastric cancer; (d) colorectal cancer; (e) cervical cancer; (f) ovarian cancer; (g) cervical cancer; (h) ovarian cancer; (i) uterine endometrium; (j) uterine myometrium. $(\mathbf{a}-\mathbf{f})$ and (i) and (j), immunostaining was visualized with a horseradish peroxidase reaction. Nestin was stained brown. (g) and (h) localization of nestin (green) and CD34 (red).

comparable to the 1iNP in U251 cells (Figure 3d, right panel).

The present luciferase assay revealed that the absolute values of luminescence were about fivefold lower in HUVECs than in U251 cells. To examine this difference, we compared each regulator activity with GFP expression using adenoviral gene transfer. In U251 cells, the proportion of GFPpositive cells was basically in parallel with the luciferase activity for each nestin regulator, and GFP-expressing cells under the 2iNP regulator were a little fewer in number than those under the CAG promoter (Figure 4, upper panel). In HUVECs, however, there were markedly fewer GFP-positive cells under nestin regulators than those under the CAG promoter, although the number of GFP-positive cell number was highest under the 1iNP regulator (Figure 4, lower panel). Given the strong nestin 


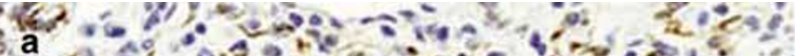

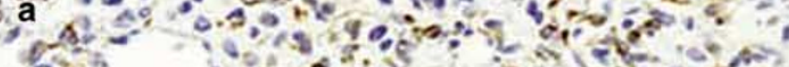

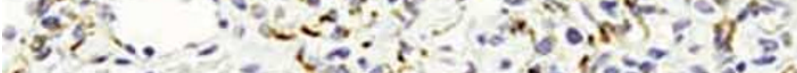

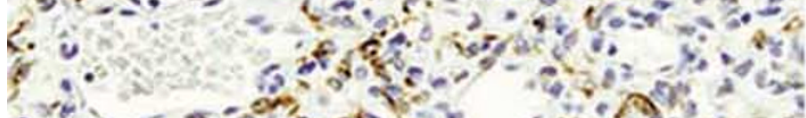
- $>$ - कe

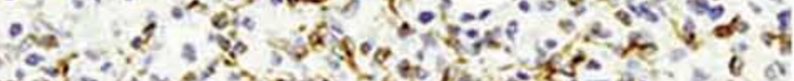

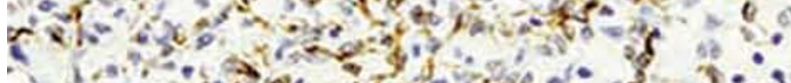

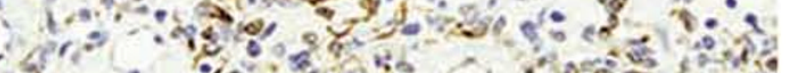

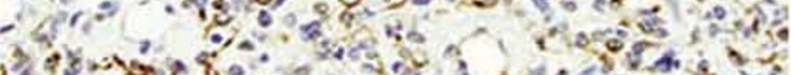
1.67\%

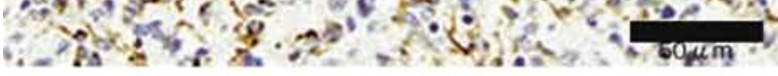

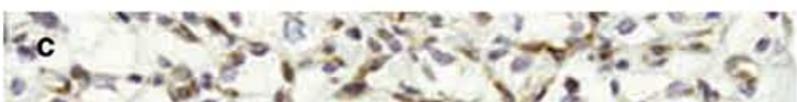
Q

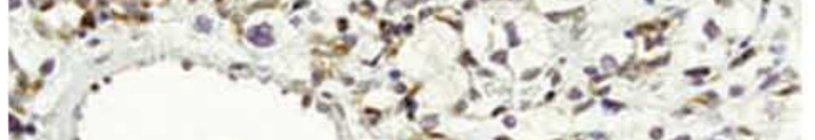

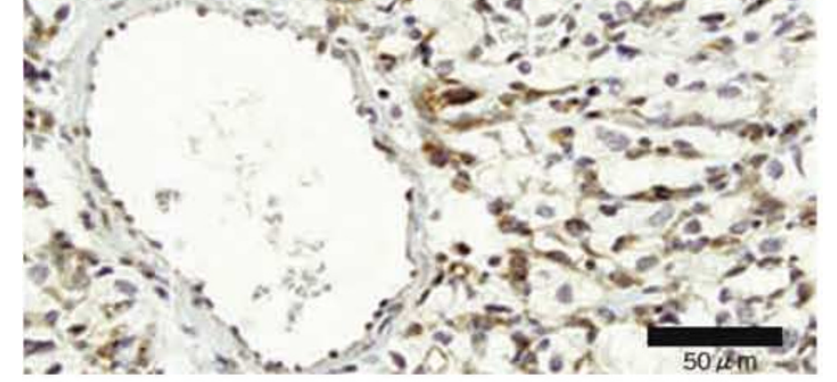

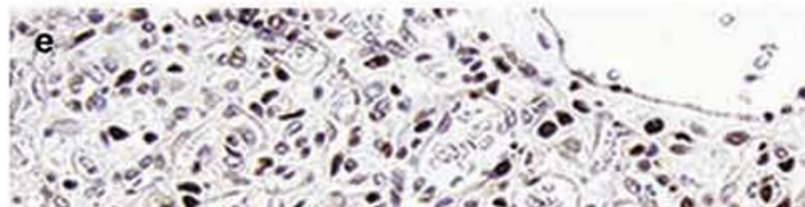

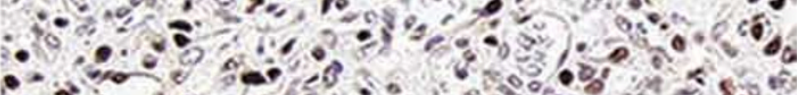

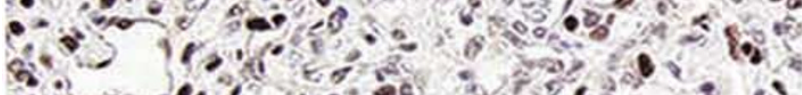

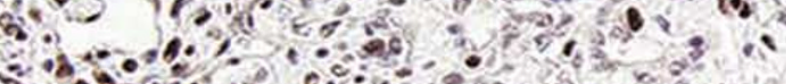

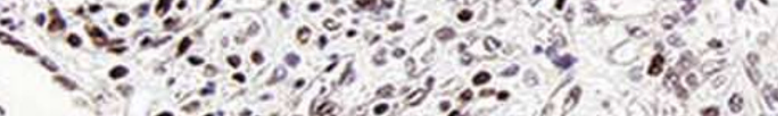

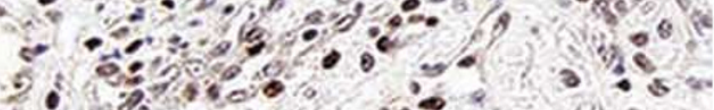
PQS

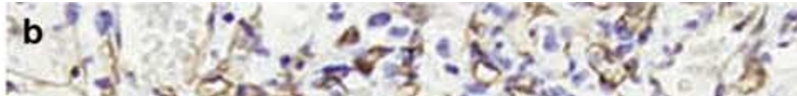

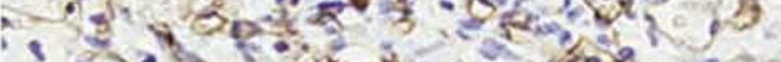
a.

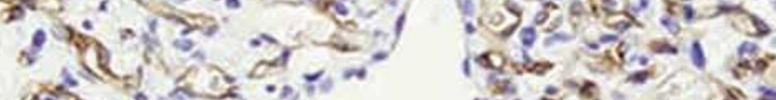

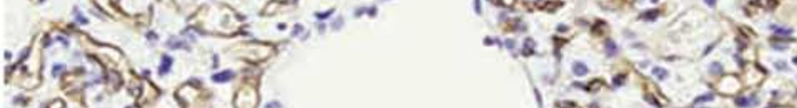

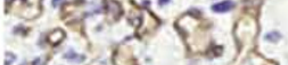



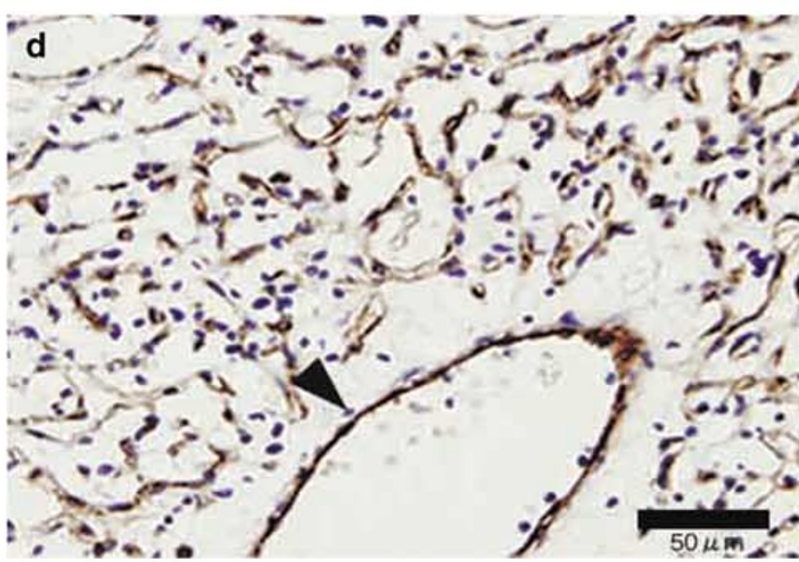
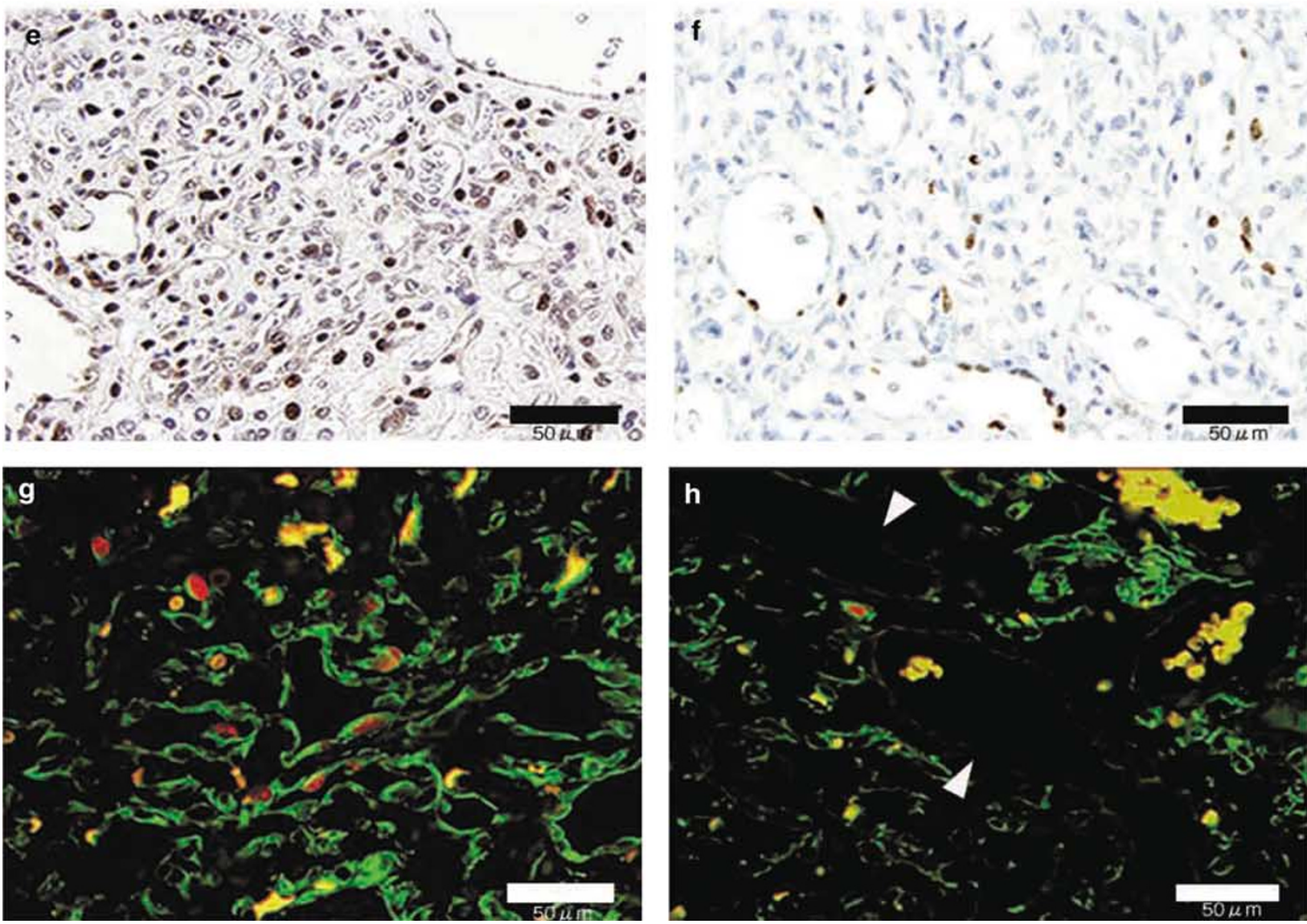
a

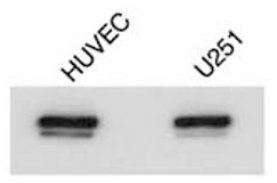

b
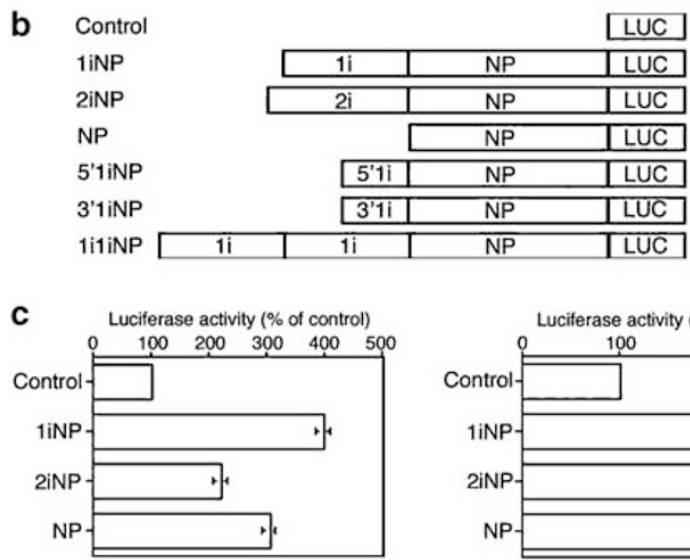

d Luciferase activity (\% of control)
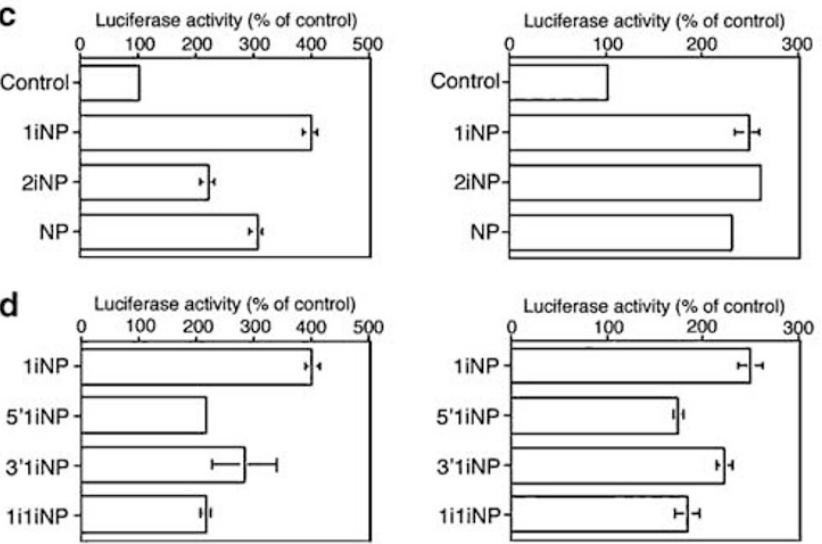

Figure 3 Luciferase assay under nestin gene regulators. (a) Western blot of nestin in HUVECs and U251 cells. (b) Structure of nestin regulators. Six nestin regulators were fused to the firefly luciferase gene. (c) luciferase activity under the three nestin regulators, 1iNP, 2iNP, and NP. The data are presented as X-fold increases against the promoterless luciferase construct. (d) luciferase activity under another three nestin regulators, 5'1iNP, $3^{\prime} 1 \mathrm{iNP}$, and 1i1iNP.

expression in HUVECs (Figure 3a), we were surprised to find that the nestin regulators were not active in HUVECs, unlike those in U251 cells. To investigate this in more detail, we further studied the nestin expression by in situ hybridization.

\section{In situ Hybridization for Nestin mRNAs in HUVECs}

By the antisense probe, nestin mRNA-positive cells were found to be scattered in the manner of the GFPpositive cells in HUVECs with a confluent density (Figure 5a, left). In contrast, the staining was noted over all the U251 cells even if the cells were in confluent culture (Figure 5a, right). No staining was noted by the sense probe (Figure 5a, lower panel). The ratio of nestin mRNA-positive cells increased in
HUVECs with less than $50 \%$ cell density culture (Figure 5b). We then re-examined the relation between cell number and GFP-positive cells under 1iNP regulator in Figure 4. The ratio of GFP-positive cells to total cells increased as the cell density decreased (Figure 5c). Since nestin was well stained in proliferative microcapillary cells in hemangioblastomas, it is reasonable that nestin mRNA was more abundant in HUVECs at a replication stage, and declined with growth arrest by contact inhibition. In contrast, the U251 cells were observed to actively replicate without growth arrest. As a reference for proliferation-independent expression, we examined $\beta$-actin mRNA expression in HUVECs, which stained evenly irrespective of cell density (data not shown). Thus, nestin mRNA expression appears to be proliferation-dependent in endothelial cells.

\section{Effect of Nestin Regulators on Tubular Network Formation}

We also examined the nestin expression in tubular network-formed endothelial cells on Matrigel culture, in which HUVECs display a tube-like structure in a VEGF-dependent manner (Figure 6A-a and -b), and nonintegrated cells to tubular network undergo apoptosis. ${ }^{15}$ Because no fluid is running in the tubular structure, the tubular form may correspond to microcapillary vessels in hemangioblastomas. ${ }^{9}$ We used $\Delta \mathrm{KDR}$ as a reporter ${ }^{17}$ so that the $\Delta \mathrm{KDR}-$ positive cells could easily undergo apoptosis without tubular formation. Interestingly, the tubular network was extensively inhibited when $\Delta \mathrm{KDR}$ was expressed under the 1iNP regulator (Figure 6A-c). An inhibitory effect of $\triangle \mathrm{KDR}$ on the tubular network formation was less evident under the NP regulator (Figure 6A-e), and least evident under the 2iNP (Figure 6A-d). In consistent with an inhibitory activity of $\triangle \mathrm{KDR}$ by nestin promoters, $\triangle \mathrm{KDR}$ expression was the highest under the 1iNP in HUVECs remained on a plastic plate (Figure 6B). Thus, we suggest that the proliferating endotheliumspecific nestin regulator is localized mostly in the first intron, and partly in the $5^{\prime}$ upstream region of the gene.

\section{Discussion}

Because tumor epithelium is characterized by neoangiogenic growth, ${ }^{12,14}$ we presumed that nestin expression is specific for angiogenic endothelial cells. Indeed, nestin is extensively expressed in developing blood vessels in a variety of tissues

Figure 2 Immunostaining of nestin, hemangioblast markers CD34 and KDR, and proliferation markers PCNA and Ki-67, in hemangioblastomas. (a) nestin; (b) CD34; (c) KDR; (d) vWF; (e) PCNA; (f) Ki-67; (g) and (h) double immunostaining of nestin (green) and Ki-67 (red). (a-f) nestin was visualized brown. Arrowhead in (d) indicates vWF staining along mature-type endothelium. Note that mature-type endothelium is negative for nestin, CD34, and KDR. (g) and (h) yellow is aggregated red blood cells. Arrowheads in (h) indicate nestin-negative mature-type endothelium. 

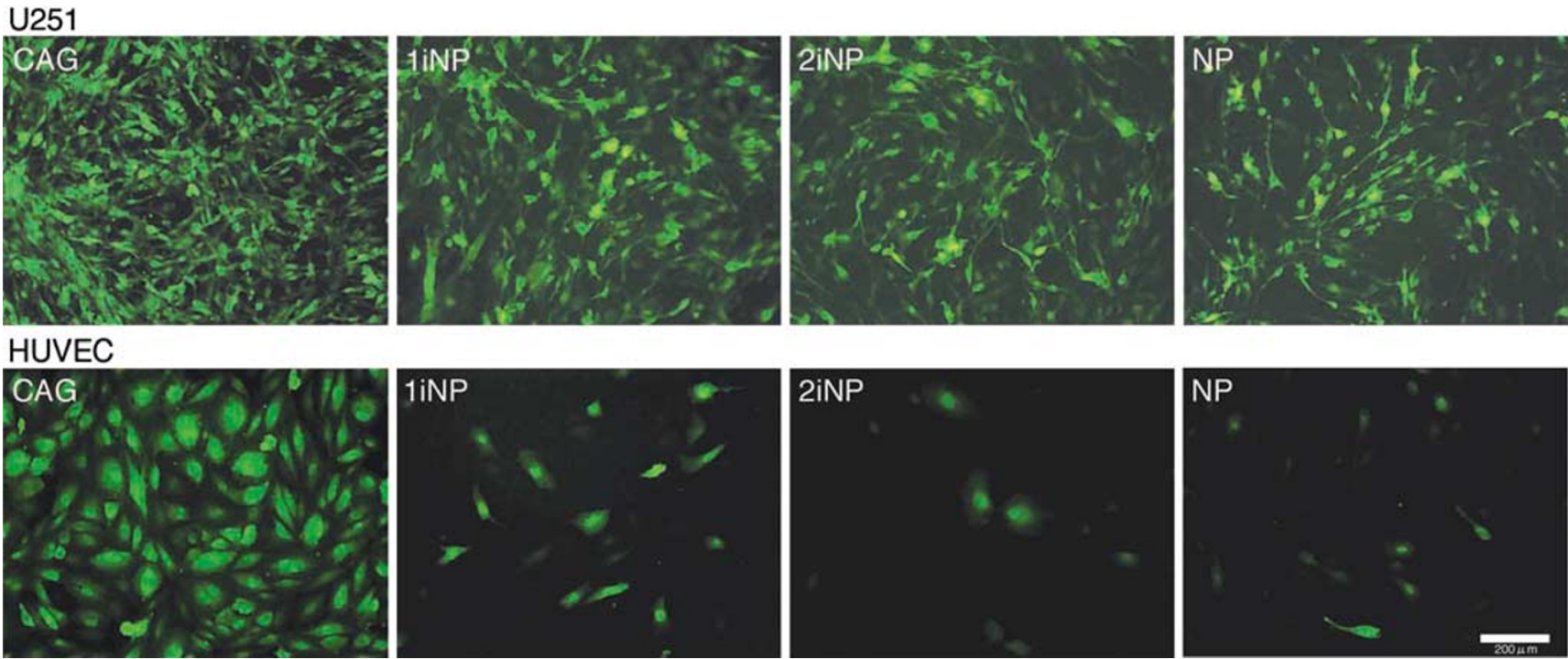

Figure 4 GFP fluorescence under the nestin gene regulators. Upper panels, U251 cells; lower panels, HUVECs. As a positive control, GFP was expressed under the CAG promoter.

including the placenta in the rat fetus. ${ }^{22}$ In this study, we examined the expression in common tumors such as lung cancers, gastrointestinal cancers, hepatocellular carcinomas, uterine cancers, and ovarian cancers. As expected, nestin-positive intratumor endothelium was detected in $79 \%$ of 82 common tumors (Table 1). Furthermore, nestinpositive endothelium was noted in the uterine endometrium and myometrium, suggesting that active angiogenesis is periodically underway in both.

Nestin-positive intratumor endothelial cells are presumed to be hemangioblast-like cells. ${ }^{13}$ Since this cell line is not available, we analyzed nestinexpressing cells in hemangioblastomas, where the primary tumorigenic cells are stromal cells which express pVHL, a proteasome-associated protein responsible for the degradation of hypoxia-inducible factors. ${ }^{23}$ However, the mitotic rate of stromal cells with the Ki-67 index is reportedly as low as less than $1 \% .^{20,21}$ In contrast, Ki-67-positive cells were noted in microcapillary endothelial cells, although they were fewer in number than another proliferation marker, PCNA-positive cells (Figure $2 \mathrm{e}$ and f). ${ }^{16}$ Many nestin-positive cells do not always express Ki-67, but nestin is always expressed in Ki-67positive cells and Ki-67 was barely present in nestin-negative cells (Figure $2 \mathrm{~g}$ and $\mathrm{h}$ ). Nestin was densely positive through the whole microcapillary region, as were CD34, KDR, and PCNA (Figure 2). Thus, nestin is positively expressed in microcapillary cells for some time after the replication, and disappears with differentiation.

To identify a tissue/cell-specific promoter/enhancer of the gene, the luciferase activity may be evaluated as a reporter using a cell line with celltype specificity. We used this assay under several nestin gene regulators with HUVECs in comparison with U251 cells. The luciferase assay values using HUVECs were about five-fold lower than that using U251 cells, although the luciferase activity was highest with the nestin regulator 1iNP. An adenovirus vector-integrated GFP expression revealed that GFP-positive HUVECs were scattered among the GFP-negative cells unlike evenly distributed GFPpositive U251 cells over a culture plate. Thus, although nestin protein levels were similar between the two cell lines, the expression of reporters was quite limited in HUVECs.

The nestin mRNA expression was also limited to a few number of HUVECs with a confluent density culture, as was the distribution of GFP-positive HUVECs. However, the proportion of the mRNApositive cells increased as the cultured cell density decreased. In contrast, ubiquitous nestin mRNA staining was observed over all the U251 cells even in confluent culture. Thus, HUVECs appears to exhibit nestin mRNA expression in a proliferation-dependent manner, because they show cell growth arrest when they reach high cell density unlike U251 cells. Indeed, we previously showed that bovine aortic endothelial cells express a high level of nestin in static culture when proliferating, while they lose nestin expression under a physiological level of shear stress flow for $12 \mathrm{~h} .{ }^{9}$ A physiological level of laminar flow is known to induce growth arrest and endothelial quiescence. ${ }^{10}$ Furthermore, the present study showed that nestin was well stained in proliferation-associated proteins, as revealed by PCNA- and Ki-67-positive areas in hemangioblastomas. In neural and glial cells, nestin is expressed in BrdU-incorporated neural progenitor cells. ${ }^{24}$ Nestin reportedly induces disassembly of vimentin to nonfilamentous aggregates or particles in mitotic 
a

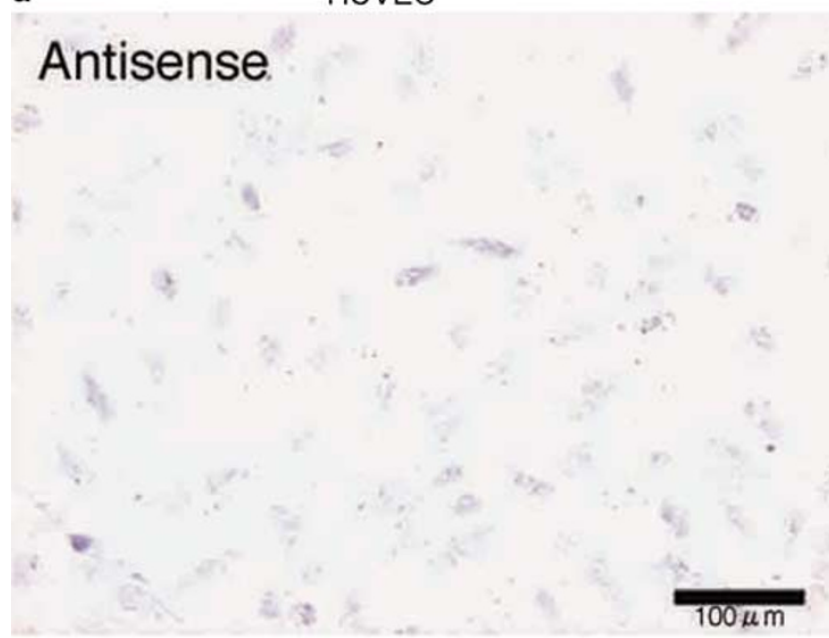

Sense

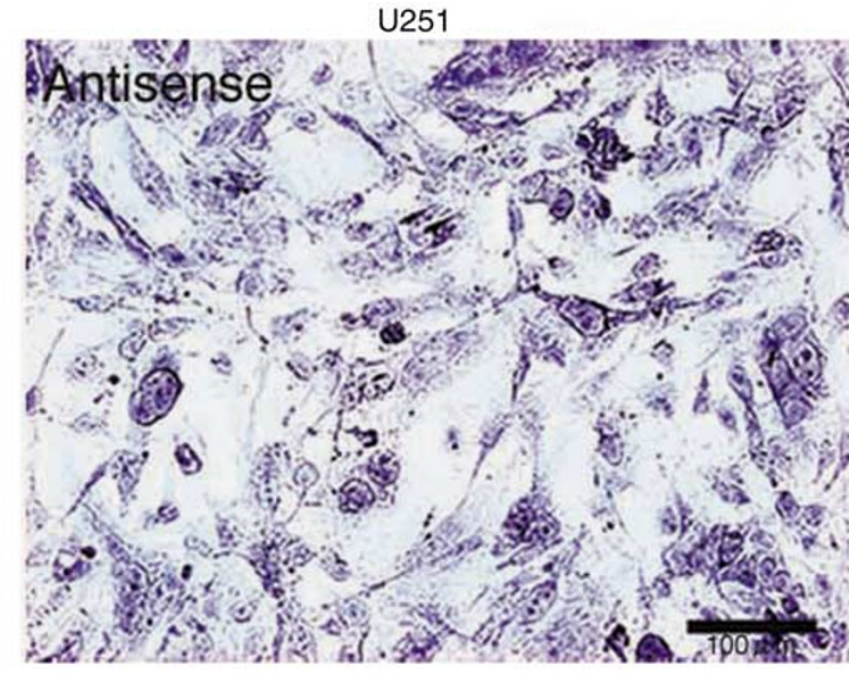

Sense
$100 \mu \mathrm{m}$

b

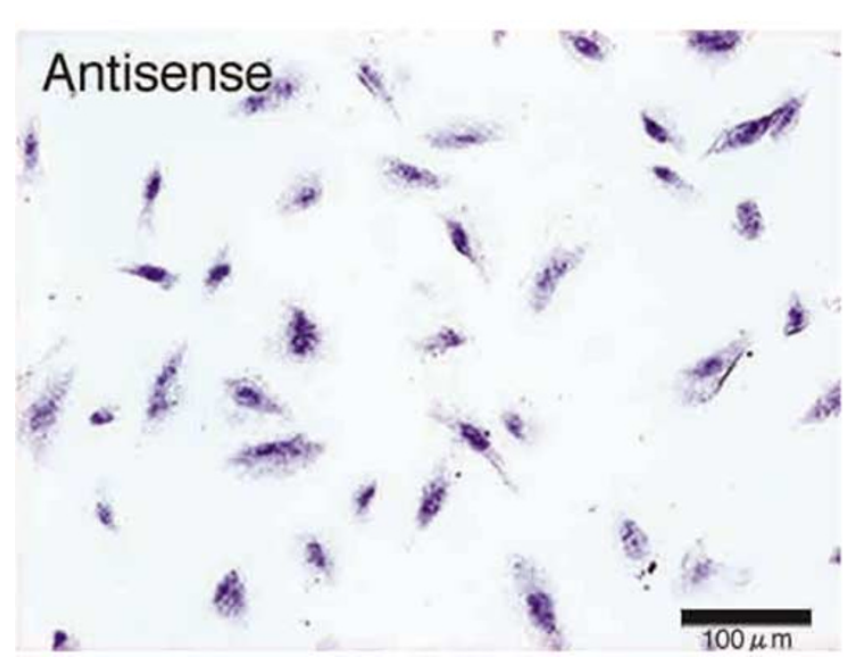

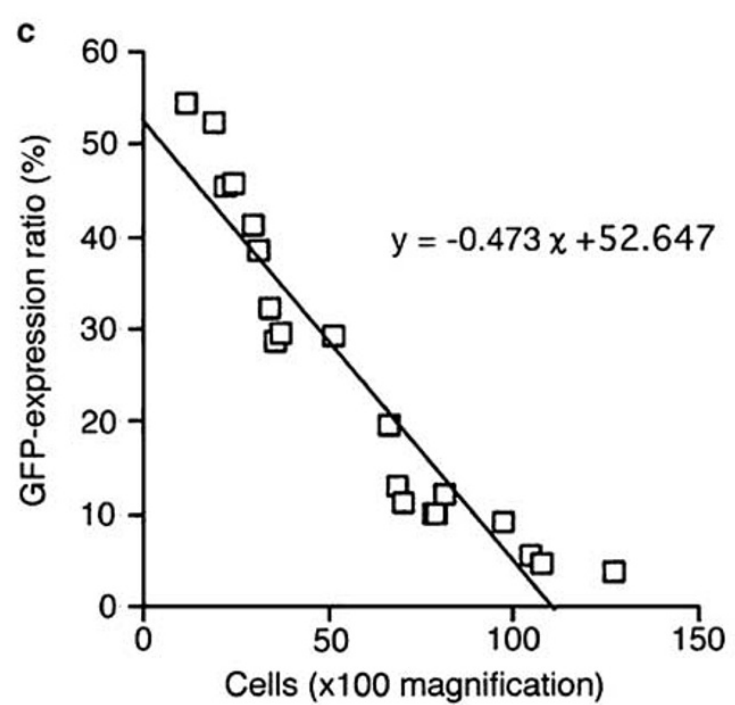

Figure 5 In situ hybridization of nestin mRNA. (a) Left panel, HUVECs; right panel, U251 cells. Cells were confluent over a plastic plate. (b) HUVECs with approximately $50 \%$ confluent cell density. (c) the relationship between the ratio of GFP-positive cells to total HUVECs and cell density. The GFP was expressed under the 1iNP regulator.

cells for equal distribution of vimentin to daughter cells. $^{4}$ Thus, nestin expression appears to be proliferation-dependent in endothelium.
HUVECs exhibit a specific feature to form capillary-like tubes in a VEGF-dependent manner when plated on a three-dimensional Matrigel 

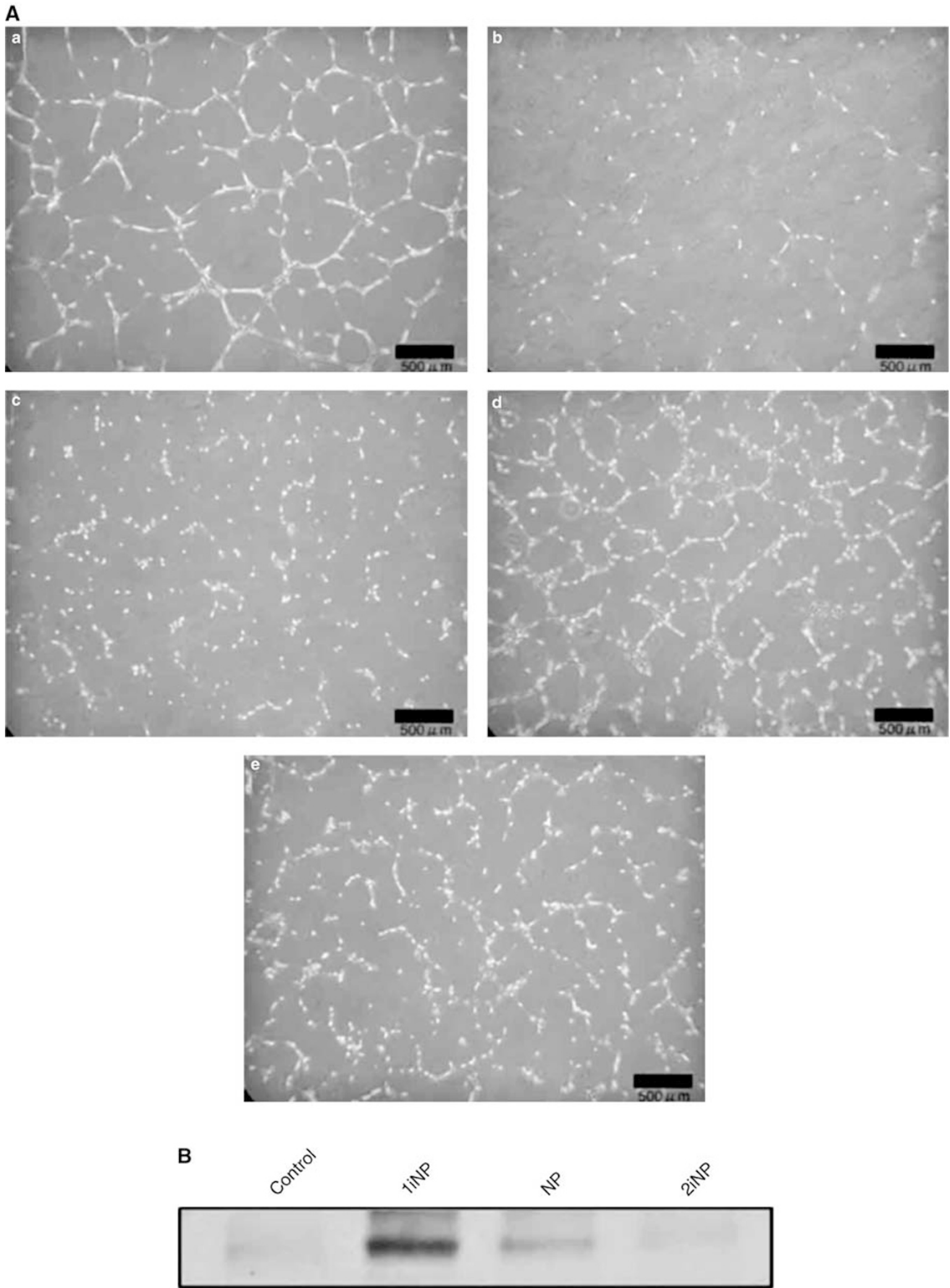

Figure 6 Effect of $\triangle K D R$ on tubular network formation under the three nestin regulators. (A) tubular network formation in Matrigel under the three nestin regulators. a-tubular network formation with VEGF at $10 \mathrm{ng} / \mathrm{ml}$. b-tubular network formation without VEGF. cinhibitory effect of $\Delta$ KDR under the 1iNP regulator. $d$-inhibitory effect of $\Delta K D R$ under the 2iNP regulator. Note that 2 iNP was least effective for the inhibition of network formation. e-inhibitory effect of $\Delta$ KDR under the NP regulator. Network formation was partially inhibited with the NP regulator. (B) Western blot of $\triangle$ KDR in HUVECs under the three nestin regulators. 
layer. In tubular network formation, HUVECs with tubular connection can survive in the Matrigel, but cells lacking a tubular connection are eliminated by apoptosis. ${ }^{15}$ Because endothelial cells without a tubular connection are easily susceptible to apoptosis, we used a tyrosine kinase domain-deleted KDR, $\Delta \mathrm{KDR} .{ }^{18}$ In our tube formation assay, the most effective regulator was 1iNP, while 2iNP and NP were less effective (Figure 6A). Thus, we suggest that the first intron contains an angiogenic cell-specific enhancer.

Intronic enhancers are not rare in intermediate filament genes including peripherin and GFAP. ${ }^{25,26}$ Peripherin is found in differentiated neurons, and the first intron has been shown to confer neuronal specificity even to heterologous HSV-TK promoter. ${ }^{25}$ In GFAP, a negative regulator was found to locate in the first intron of the gene. ${ }^{26}$ The neural stem cellspecific enhancer of the nestin gene was narrowed down to $636 \mathrm{bp}$ in rats and $714 \mathrm{bp}$ in humans in the second intron. ${ }^{2}$

An angioblast and endothelial cell-specific enhancer has been reported for the first intron of the KDR gene. ${ }^{27}$ The $3^{\prime}$ half of the first intron itself conferred the endothelium-specific expression even to the heterologous HSV-TK promoter-directed LacZ reporter expression in transgenic mice. Although the $5^{\prime}$ upstream basic promoter of the KDR gene did not give endothelium-specific expression, it contributed to a strong and uniform transgene expression, which was stimulated by the transcription factor HIF- $2 \alpha$ (also known as HLF, or EPAS1). ${ }^{27}$ The HIF- $2 \alpha$ is known to be induced by hypoxia and degraded by the pVHL degradation system in endothelial cells while HIF- $1 \alpha$ is known to stimulate VEGF gene expression in tumor cells. ${ }^{23}$ The endothelium-specific region of the KDR first intron was narrowed to the $510 \mathrm{bp}$ sequence before the second exon, which contains potential binding sites for the GATA, a GATA-cored transcription factor, and Ets transcription factor, and for Scl/Tal-1. ${ }^{27}$ We searched potential transcription factor-binding sites in the first intron of the nestin gene, and found at least three potential binding sites for GATA and one putative enhancer element $5^{\prime}$-CACGTG-3' for HIF- $2 \alpha$.

Endothelial cells can be derived from several different sources: bone marrow stromal stem cells, pericytes, and pre-existing endothelial cells. Bone marrow-derived endothelial progenitor cells have recently been shown to be integrated in neoangiogenic endothelium in tumors. ${ }^{12}$ Nestin is extensively expressed in these newly forming angiogenic components including endothelial precursor cells, pericytes, and microvessel-associated mesenchymal cells. ${ }^{28}$ We suggest that the nestin first intron-directed expression of cytocidal genes in endothelial precursor cells may be useful for developing an antiangiogenic cancer therapy.

\section{Acknowledgements}

We thank Ms M Kosaki and Ms M Hosoi for their secretarial and technical assistances. This work was supported by grants-in-aids and the 21st Century COE Program from the Japanese Ministry of Education, Culture, Science, Sports, and Technology.

\section{References}

1 Lendahl U, Zimmerman LB, Mckay RDG. CNS stem cells express a new class of intermediate filament protein. Cell 1990;60:585-595.

2 Johansson CB, Lothian C, Molin M, et al. Nestin enhancer requirements for expression in normal and injured adults CNS. J Neurosci Res 2002;69:784-794.

3 Eliasson C, Sahlgren C, Berthold C-H, et al. Intermediate filament protein partnership in astrocytes. J Biol Chem 1999;274:23996-24006.

4 Chou Y-H, Khuon S, Herrmann H, et al. Nestin promotes the phosphorylation-dependent disassembly of vimentin intermediate filaments during mitosis. Mol Biol Cell 2003;14:1468-1478.

5 Zimmerman L, Lendahl U, Cunningham $\mathrm{M}$, et al. Independent regulatory elements in the nestin gene direct transgene expression to neural stem cells or muscle precursors. Neuron 1994;12:11-24.

6 Keyoung HM, Roy NS, Benraiss A, et al. High-yield selection and extraction of two promoter-defined phenotypes of neural stem cells from the fetal human brain. Nat Biotechnol 2001;19:843-850.

7 Dahlstrand J, Collins VP, Lendahl U. Expression of the class VI intermediate filament nestin in human central nervous system tumors. Cancer Res 1992;52: 5334-5341.

8 Kurihara H, Zama A, Tamura M, et al. Glioma/ glioblastoma-specific adenoviral gene expression using the nestin gene regulator. Gene Therapy 2000;7: 686-693.

9 Sugawara K, Kurihara H, Negishi M, et al. Nestin as a maker for proliferative endothelium in gliomas. Lab Invest 2002;82:345-351.

10 Malek AM, Alper SL, Izumo S. Hemodynamic shear stress and its role in atherosclerosis. JAMA 1999;282: 2035-2042.

11 Lothian C, Lendahl U. An evolutionarily conserved region in the second intron of the human nestin gene directs gene expression to CNS progenitor cells and to early neural crest cells. Eur J Neurosci 1997;9:452-462.

12 Rafii S, Lyden D, Benezra R, et al. Vascular and haematopoietic stem cells: novel targets for antiangiogenesis therapy? Nat Rev Cancer 2002;2:826-835.

13 Pelosi E, Valtieri M, Coppola S, et al. Identification of the hemangioblast in postnatal life. Blood 2002; 100:3203-3208.

14 Lyden D, Hattori K, Dias S, et al. Impaired recruitment of bone-marrow-derived endothelial and hematopoietic precursor cells blocks tumor angiogenesis and growth. Nat Med 2001;7:1194-1201.

15 Pollman MJ, Naumovski L, Giboons GH. Endothelial cell apoptosis in capillary network remodeling. J Cell Physiol 1999;178:359-370.

16 Iwata K, Nakagawa H, Hashizume Y. Significance of MIB-1, PCNA indices, and p53 protein over-expression 
in intramedullary tumors of the spinal cord. Brain Tumor Pathol 1996;13:73-78.

17 Niwa H, Yamamura K-I, Miyazaki J-I. Efficient selection for high-expression transfactants with a novel eukaryotic vector. Gene 1991;108:193-200.

18 Millauer B, Shawver LK, Plate KH. Glioblastoma growth inhibited in vivo by a dominant-negative Flk1 mutant. Nature 1994;367:576-579.

19 Kim HS, Kang HS, Messam CA, et al. Comparative evaluation of angiogenesis in gastric adenocarcinoma by nestin and CD34. Appl Immunohistochem Mol Morphol 2002;10:121-127.

20 Böhling T, Plate KH, Haltia MJ, et al. Von HippelLindau disease and capillary haemangioblastoma. In: Kleihues P, Cavenees WK, (eds). Pathology and Genetics of Tumours of the Nervous System. IARC Press: Lyon, 2000, pp 223-226.

21 Lach B, Gregor A, Rippstein P, et al. Angiogenic histogenesis of stromal cells in hemangioblastomas: ultrastructural and immunohistochemical study. Ultrastruct Pathol 1999;23:299-310.

22 Mokry J, Nemecek. Angiogenesis of extra- and intraembryonic blood vessels is associated with expression of nestin in endothelial cells. Folia Biol (Prague) 1998;44:155-161.

23 Brahimi-Horn C, Berra E, Pouysségur J. Hypoxia: the tumor's gateway to progression along the angiogenic pathway. Trends Cell Biol 2001;11:S32-S36.

24 Messam CA, Hou J, Major EO. Coexpression of nestin in neural and glial cells in the developing human CNS defined by a human-specific anti-nestin antibody. Exp Neurol 2000;161:585-596.

25 Lecomte M-J, Basseville M, Fauquet M. Involvement of intronic sequences in cell-specific expression of the peripherin gene. J Neurochem 1999;73:1806-1815.

26 Sarkar S, Cowan NJ. Intragenic sequences affect the expression of the gene encoding glial fibrillary acidic protein. J Neurochem 1991;57:675-684.

27 Kappel A, Rönicke V, Damert A, et al. Identification of vascular endothelial growth factor (VEGF) receptor-2 (Flk-1) promoter/enhancer sequences sufficient for angioblast and endothelial cell-specific transcription in transgenic mice. Blood 1999;93:4284-4292.

28 Lardon J, Rooman I, Bouwens L. Nestin expression in pancreatic stellate cells and angiogenic endothelial cells. Histochem Cell Biol 2002;117:535-540. 